\title{
CMS Conference Report
}

October 4, 2006

\section{The CMS Forward Pixel Detector}

\author{
Sudhir Malik \\ Univeristy of Nebraksa - Lincoln, 116 Brace Lab, Lincoln, Nebraska, USA \\ CMS Collaboration
}

\begin{abstract}
At the core of CMS all-silicon tracker is the pixel detector. It provides fine granularity for charged track reconstruction with three-dimensional space measurements with resolutions on the order of ten microns. It is comprised of two parts - the barrel detector and the forward disks. The disks are called the Forward Pixel detector and are being assembled at Fermilab, U.S.A. The detector modules for the disks consist of eighteen million pixels and the production begins in summer of 2006. The designs and the present status of the detector are reviewed here.
\end{abstract}

\author{
Keywords: CMS; silicon tracker; pixel detector; radiation hardness; detector assembly \\ Presented at: \\ X Pisa Meeting, Frontier Detectors for Frontier Physics, \\ La Biodola, Isola d'Elba, Italy, May 21-27, 2006
}

To appear in Conference Proceedings in Nuclear Instrumentation and Methods 


\section{Introduction}

The CMS (Compact Muon Solenoid) experiment [1] is a major collider detector experiment being built to utilize the LHC (Large Hadron Collider). It will detect the proton-proton collisions at $14 \mathrm{TeV}$. A robust tracking combined with a precise vertex reconstruction within a strong magnetic field of 4 Tesla will play a key role to address the full range of physics that would be accessible at this energy. This is realized by using an all-silicon tracking system [2] as the innermost component of the CMS detector. At the core of this system lies the pixel detector [3]. The pixel detector consists of two components - the barrels and the disks. There are 3 layers of barrels complemented with 2 disks on each side. The disks are called the Forward Pixel detector. Innovative techniques of radiation hard technology and bump bonding have been used to meet the challenge of high radiation environment and small space availability. The pixel detector is expected to provide a hit resolution of $10 \mu \mathrm{m}$ in the $(\mathrm{r} \phi)$ and $17 \mu \mathrm{m}$ in $(\mathrm{r} \eta)$ coordinates and remain functional up to a fluence of $6 \times 10^{14} \mathrm{n}_{\mathrm{eq}} / \mathrm{cm}^{2}$.

\section{The Detector Layout}

The Forward Pixel Detector is a very sophisticated device and consists of 2 disks (see Figure 1) on each side of the pixel barrel at a distance of $34.5 \mathrm{~cm}$ and $46.5 \mathrm{~cm}$ from its center. These disks are populated with 672 detector modules called plaquettes. A plaquette is a multilayered structure assembled in three steps. First, an array of readout chips (ROCs) is bump bonded to a single silicon sensor and forms a bump bonded module. Then the bump bonded module is glued to a flex circuit called Very High Density Interconnect (VHDI) laminated on a silicon substrate. Finally, pads on ROCs and VHDI are connected through wire bonds. There are five different kinds of plaquettes depending on the number of ROCs varying from two to ten. Plaquettes are precisely glued and wire bonded in groups of three or four on another flex circuit called High Density Interconnect (HDI) that has been laminated to a thin wedge shape beryllium piece and forms a panel. A panel also carries a Token Bit Manager (TBM) [4] that orchestrates the readout from the ROCs. TBM is also wire bonded to the HDI. A panel is mounted on each of the two sides of a V-shaped aluminum cooling channel and forms a blade. One panel on a blade carries four plaquettes and faces the interaction region while the other has three plaquettes. The positioning of plaquettes on panels is constrained by the need to cover up the gaps between the plaquettes. There are 24 blades that are arranged in a turbine like circular geometry and form a disk. Each blade is rotated by $20^{\circ}$ around its axis of radial symmetry. This rotation enhances the spatial resolution by charge sharing among neighboring pixels. The disk is annular in structure with an inner and an outer radius of $60 \mathrm{~mm}$ and $150 \mathrm{~mm}$ respectively. This allows a coverage in pseudo rapidity $(\eta)$ from 1.5 to 2.25 .
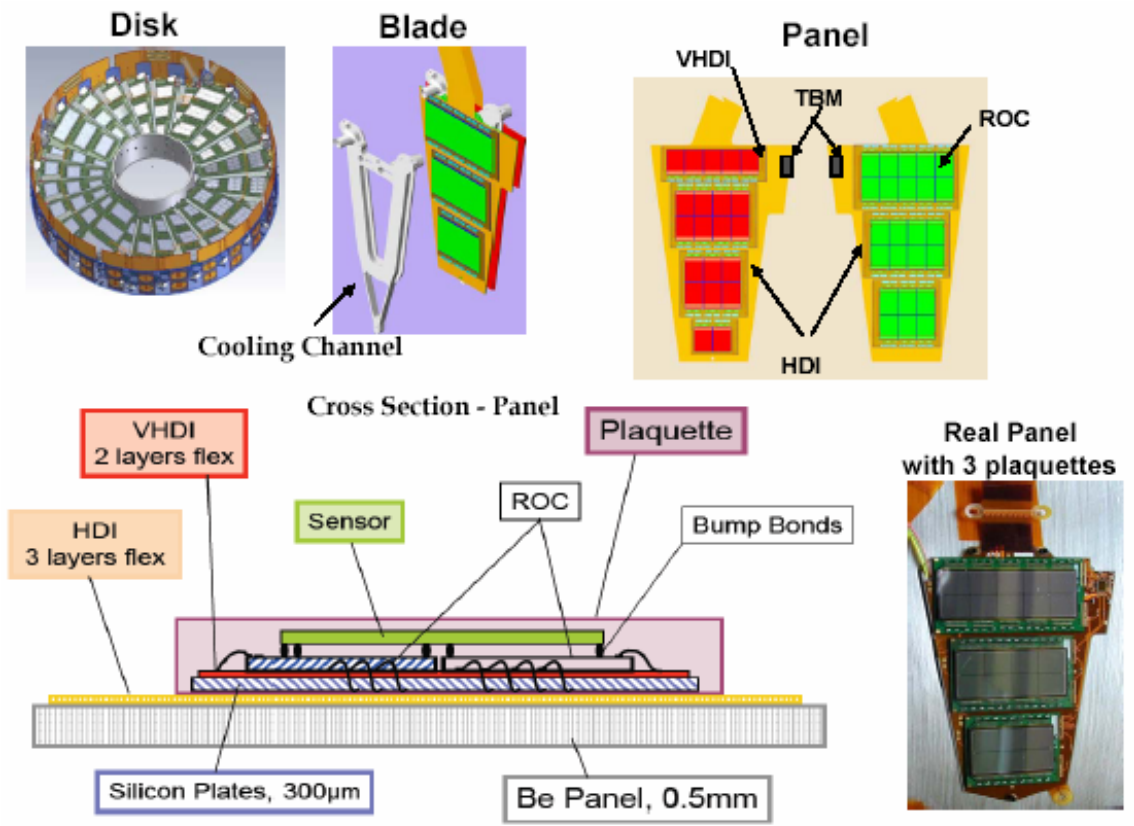

Figure 1: The disks are a complex structure. They are made of plaquettes, panels and blades. Plaquettes are multilayered structure of different sizes. 
The sensor [5,6] and the ROC are designed and well tested to be radiation tolerant for the LHC environment. The sensor design is of $\mathrm{n}^{+}$on $\mathrm{n}$-type. A p-stop isolation technique in which a high dose $\mathrm{p}$-implant surrounds each $\mathrm{n}+$-type region provides explicit electrical isolation between neighboring $\mathrm{n}+$ electrodes. Each sensor is $300 \mu \mathrm{m}$ thick and consists of an array of pixels of size $100 \mu \mathrm{m} \times 150 \mu \mathrm{m}$ in two dimensions.

The ROC has been fabricated in $0.25 \mu \mathrm{m}$ CMOS technology [7]. To align and bump bond with the sensor pixels, each ROC consists of an array of 4160 pixels arranged in 52 columns and 80 rows. A total of 4320 ROCs needed to construct four disks. This puts the number of channels to be readout around 18 million.

An optical readout is used to reduce electrical interference among the analog signals. The TBM on each panel organizes the readout in the front end. Through a token bit mechanism it controls the access of each column in a ROC to the readout link. It also formats the data packets and signals any errors. The data is readout in two steps [8]. When a pixel is hit the corresponding information about its time, address and analog signal is stored in the ROC. In the next step, when a trigger arrives, a token is generated and passed to all the ROCs on a panel. Then the analog data of the triggered event stored in the ROC is read out. The analog signal is converted to an optical signal using an opto-hybrid and is sent via optical links to the counting room. Here a front-end digitizer converts the signal back from optical to electrical, performs the digitization, formats the event and sends it to CMS DAQ event builder..

\section{Detector Assembly and Construction}

Tremendous progress has been made in the past few months towards the final assembly and construction of the detector at Fermilab. All the components (sensors, ROCs, Be panels, VHDI, HDI) to begin the production are in hand Final assembly begins summer of 2006 and shall last ten months. Testing and assembly of some of the components required for the final assembly are being done at collaborating institutions other than Fermilab. The production is broadly divided into two parts - plaquette and panel. In order to qualify and mount a plaquette on a panel it is required to fully test and characterize it. To achieve this goal, special electronic setups have been designed and are capable of testing the plaquettes and panels at a temperature of $-20^{\circ} \mathrm{C}$. Fast custom built software has been developed to perform all the characterization tests quickly and store the data produced in a database for a later retrieval during commissioning and calibration phase. These have been thoroughly tested using pre-production plaquettes and panels. We have the capability to completely test 6 plaquettes and two panels in a day.

The plaquettes would undergo several burn-in thermal cycles from $-20^{\circ} \mathrm{C}$ to $+20^{\circ} \mathrm{C}$ before they are tested in detail. This is required in order to make sure that only those plaquettes that survive thermal stress get tested and mounted. As the panels get tested successfully they will be mounted on the half-disks. The half disks and the final electronics would then be mounted on the half-cylinder for a system test.

There has been a tremendous progress with respect to procuring and testing final electronic components, developing slow controls for the system and finalizing the mechanical details of detector installation at CERN. The software tools for detector commissioning, calibration, operations and data quality monitoring are also being developed in parallel. Prototype version of some of them exists already. The geometry of the disks is also complete and has been incorporated into the full GEANT simulation of the CMS detector.

\section{Irradiation and Test Beam Efforts}

In the last few months a considerable effort has been spent in irradiating the pre-production detector components at the Indiana University Radiation Facility (IUCF) and then taking data with them in a real beam environment at Fermilab. Several plaquettes have been irradiated up to a proton fluence of $1.3 \times 10^{14} / \mathrm{cm}^{2}$ in September 2005 at IUCF, along with TBM chip, prototype adaptor board, temperature sensors, and other components. Earlier testbeam [9,10] runs demonstrated a high sensor efficiency and spatial resolution of $15 \mu \mathrm{m}$ achievable by rotating the detectors by $20^{\circ}$ and charge sharing. In a test beam in January 2006, data was taken using the above irradiated detectors. A thorough and detailed analysis has shown that the irradiated detectors 
work excellently and the efficiency is close to $100 \%$ across the pixel cells (see Figure 2). The very same detectors have been exposed again up to a proton fluence of $8 \times 10^{14} / \mathrm{cm}^{2}$ along with bump bonded modules, capacitors, and VHDIs laminated to silicon substrate. These detectors will be again subjected to a test beam run in July 2006 at Fermilab to see any change in their behavior at the higher dose. A visual inspection of the VHDIs shows no signs of its delamination from the silicon substrate. These efforts have proved that the sensors, ROCs and other components meet the design requirements.

RUN 7612

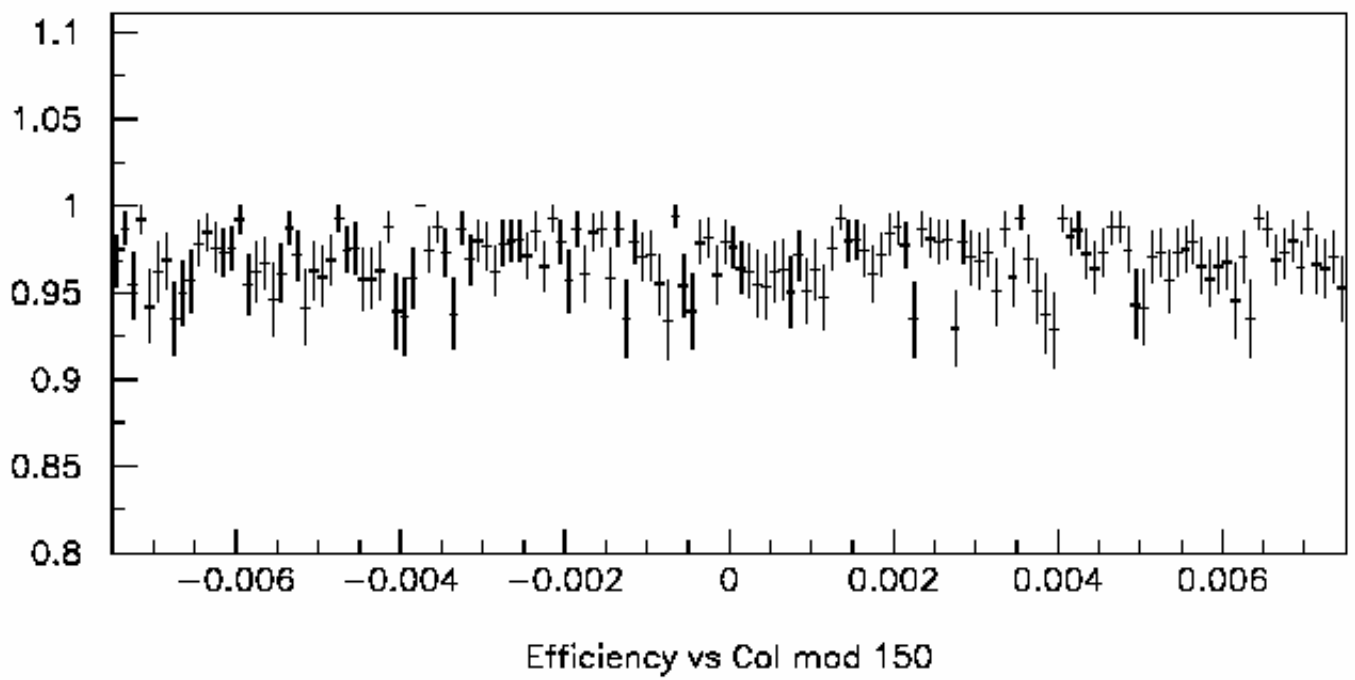

Figure 1: Particle detection efficiency across pixel cells of an irradiated plaquette.

\section{Conclusions}

Tremendous progress has taken place in the last year towards preparation of Forward Pixel Detector for production. The production starts in summer of 2006 at Fermilab. The final detector will be shipped to CERN in summer of 2007. After commissioning phase it will be integrated into the complete CMS tracker in 2008.

\section{References}

[1] The CMS Collaboration, CMS Tracker Technical Design Report, CERN/LHCC 1998-6, 1998.

[2] F. Hartmann [CMS Silicon Tracker Collaboration], Nucl. Instr. Meth. A 478, 285 (2002).

[3] D. Kotlinski, Nucl. Instrum. Meth. A 477, 446 (2002).

[4] E. Bartz, "The token bit manager chip for the CMS pixel readout". Prepared for 11th Workshop on Electronics for LHC and Future Experiments (LECC 2005), Heidelberg, Germany, 12-16 September 2005.

[5] K. Arndt et al, Nucl. Instrum. Meth.. A 511, 106(2003).

[6] G. Bolla et al, Nucl.Instrum.Meth .A 485, 89 (2002).

[7] W. Erdmann [CMS Collaboration], Nucl. Instr. Meth. A 549, 153 (2005).

[8] M Barbero et al. Published in Nucl. Instrum. Meth. A 517, 349 (2004).

[9] G. Bolla et al, Nucl. Instr. Meth. A 501, 160 (2003).

[10] M. Atac et al., Published in Nucl. Instrum. Meth.A 488, 271(2002). 